Biochem Biophys Res Commun. 2011 January 21; 404(3): 853-858. doi:10.1016/j.bbrc.2010.12.074.

\title{
Posttranslational negative regulation of glycosylated and non- glycosylated BCRP expression by Derlin-1
}

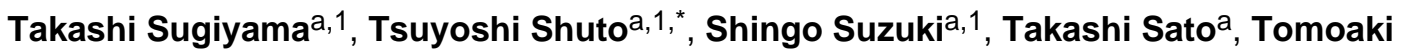 \\ Koga $^{a}$, Mary Ann Suico ${ }^{a}$, Hiroyuki Kusuhara ${ }^{b}$, Yuichi Sugiyama ${ }^{b}$, Douglas M. Cyr ${ }^{c}$, and \\ Hirofumi Kaia, ${ }^{*}$ \\ aDepartment of Molecular Medicine, Graduate School of Pharmaceutical Sciences, Global COE \\ "Cell Fate Regulation Research and Education Unit", Kumamoto University, 5-1 Oe-Honmachi, \\ Kumamoto 862-0973, Japan \\ bLaboratory of Molecular Pharmacokinetics, Graduate School of Pharmaceutical Sciences, The \\ University of Tokyo, 7-3-1 Hongo, Bunkyo-ku, Tokyo 113-0033, Japan \\ 'Department of Cell and Developmental Biology, School of Medicine, 526 Taylor Hall, University \\ of North Carolina, Chapel Hill, NC 27599-7090, USA
}

\section{Abstract}

Human breast cancer resistance protein (BCRP)/MXR/ABCG2 is a well-recognized ABC halftransporter that is highly expressed at the apical membrane of many normal tissues and cancer cells. BCRP facilitates disposition of endogenous and exogenous harmful xenobiotics to protect cells/tissues from xenobiotic-induced toxicity. Despite the enormous impact of BCRP in the physiological and pathophysiological regulation of the transport of a wide variety of substrates, little is known about the factors that regulate posttranslational expression of BCRP. Here, we identified Derlin-1, a member of a family of proteins that bears homology to yeast Der1p, as a posttranslational regulator of BCRP expression. Overexpression of Derlin-1 suppressed ER to Golgi transport of wild-type (WT) BCRP that is known to be efficiently trafficked to the plasma membrane. On the other hand, protein expression of N596Q variant of BCRP, N-linked glycosylation- deficient mutant that preferentially undergoes ubiquitin-mediated ER-associated degradation (ERAD), was strongly suppressed by the overexpression of Derlin-1, whereas knockdown of Derlin-1 stabilized N596Q protein, suggesting a negative regulatory role of Derlin-1 for N596Q protein expression. Notably, knockdown of Derlin-1 also stabilized the expression of tunicamycin-induced deglycosylated WT BCRP protein, implying the importance of glycosylation state for the recognition of BCRP by Derlin- 1 . Thus, our data demonstrate that Derlin-1 is a negative regulator for both glycosylated and non-glycosylated BCRP expression and provide a novel posttranslational regulatory mechanism of BCRP by Derlin-1.

\footnotetext{
(C) 2010 Elsevier Inc. All rights reserved.

*Corresponding authors. Address: Department of Molecular Medicine, Faculty of Life Sciences, Kumamoto University, 5-1 Oehonmachi, Kumamoto 862-0973, Japan. Fax: +81 963714405 (H. Kai). Fax: +81 963714407 (T. Shuto). tshuto@ gpo.kumamotou.ac.jp (T. Shuto), hirokai@ @po.kumamoto-u.ac.jp (H. Kai).

${ }^{1}$ These authors contributed equally to this work.
} 


\section{Keywords}

BCRP; Derlin-1; N-linked glycosylation; Posttranslational regulation; ER-associated degradation (ERAD)

\section{Introduction}

Human ATP-binding cassette $(\mathrm{ABC})$ transporters are membrane transporters that use energy from ATP hydrolysis to transport a wide variety of substrates across the cellular membrane [1]. To date, 48 members of the human $\mathrm{ABC}$ transporters genes have been identified and cloned. Of these, breast cancer resistance protein (BCRP)/MXR/ABCG2 is a wellrecognized $\mathrm{ABC}$ half-transporter that is highly expressed at the apical membrane of many normal tissues [2,3]. BCRP mediates efflux transport of a large number of endogenous and exogenous harmful xenobiotics and facilitates disposition of these substrates, which results in protecting cells/tissues from xenobiotic-induced toxicity. Recent works on the use of $A b c g 2^{-/-}$mice have further revealed that BCRP is involved in extrusion of porphyrins, vitamin $\mathrm{B}_{2}$ (riboflavin) and other vitamins (biotin, vitamin K) [4]. Moreover, BCRP was also identified as a urate secretory transporter with a common functional genetic polymorphism causing gout [5].

Despite the enormous impact of BCRP in the physiological regulation of the transport of a wide variety of substrates, overexpression of BCRP in cancer cells reduces intracellular concentration of anticancer drugs, which dampens cytotoxic effects of these drugs [6]. Consistently, it has been reported that a correlation exists between BCRP expression and patient outcome in some hematologic and solid tumors [7], suggesting that identification of the regulatory mechanisms responsible for the expression and function of BCRP may help us to circumvent BCRP-mediated drug resistance and improve anti-cancer therapies.

The expression level of BCRP protein is regulated by both transcriptional and posttranslational mechanisms. Recent reports on the posttranslational regulation of BCRP suggested that monitoring the quality of BCRP protein in the endoplasmic reticulum (ER) is critical for the regulation of BCRP expression [8]. For example, not wild-type (WT) but N596Q variant of human BCRP, in which N-linked glycosylation was predicted not to occur at all, was susceptible to ER-associated degradation (ERAD) [9]. Moreover, the certain single nucleotide polymorphism (SNP) variants of BCRP (Q141K, F208S and S441N), which protein expression was markedly low despite the functional expression of mRNA, were also degraded by ERAD [10,11]. Although ER seems to be strongly associated with the expression level of BCRP, the molecules that are critical for the control of these posttranslational regulations are yet to be fully understood.

In the present study, we first screened ER-localized E3 ubiquitin ligases and their co-factor that functions in the regulation of WT and N596Q variant of BCRP expression and identified Derlin-1, a member of a family of proteins that bears homology to yeast Der1p [12], as a negative regulator for both glycosylated and non-glycosylated BCRP expression. In addition, we demonstrated that the difference was observed between WT and N596Q variant of BCRP with respect to the mechanism underlying negative regulation of BCRP by 
Derlin-1. Our data provide a novel posttranslational regulatory mechanism of BCRP by Derlin-1.

\section{Materials and methods}

\subsection{Materials}

Reagents, antibodies, DNA constructs and RNAi constructs used in this study are described in the Supplemental data.

\subsection{Cell culture and transfection}

General method for cell culture, cycloheximide (CHX) chase and brefeldin A (BFA) wash out experiments in this study are described in the Supplemental data. Transient transfections of plasmids were performed using either Trans-IT LT1 (Mirus, Madison, WI) or Hilymax (Dojin Co. Ltd, Tokyo, Japan) by following the manufacturer's recommendations as described earlier [13,14]. For the transfection of si-RNAs, Trans-IT TKO (Mirus) was used as previously described [15]. Fifty nanomolar of si-Derlin-1 and Stealth ${ }^{\mathrm{TM}}$ RNAi negative Universal Control (control siRNA) were transfected into 90\% confluent cells to knockdown Derlin-1. The cells were harvested $48 \mathrm{~h}$ after transfection.

\subsection{SDS-PAGE, Western blotting and immunoprecipitation}

Confluent (90-100\%) cells grown on $60 \mathrm{~mm}$ dishes were used for collect the cell lysates and are subjected to SDS-PAGE, followed by Western blotting as described earlier [8]. For Nglycanases digestion, cell lysates were denatured at $37^{\circ} \mathrm{C}$ for $30 \mathrm{~min}$ and digested with Endo H (500 U) or PNGase F (500 U) at $37{ }^{\circ} \mathrm{C}$ for $90 \mathrm{~min}$. Method on immunoprecipitation with anti-GFP antibody is described in the Supplemental data.

\subsection{Immunocytochemical analysis}

Immunocytochemical analysis was performed as described previously [16]. BCRP-GFPtransfected HeLa cells were stained with anti-KDEL antibody and Alexa Flour 546 conjugated secondary antibody (Molecular Probes, Eugene, OR).

\subsection{Statistical analysis}

For quantitative analysis, result represents the mean \pm SEM performed in triplicate and the data were analyzed by Student's $t$-test (JMP software, SAS Institute).

\section{Results}

\subsection{Screening of ER-localized factors that function in the regulation of WT and N596Q variant of BCRP expression}

Although the posttranslational modification and ERAD of BCRP are becoming of great interest [8], there is only limited information on the specific factors that are critical for the regulation of BCRP expression. In the present study, we focused on WT and N596Q variant of BCRP. WT BCRP was used as a representative of BCRP proteins that is efficiently glycosylated and folded in the ER and transported to the plasma membrane (PM) [8]. Consistently, our analysis of the expression pattern of WT BCRP in HEK293 cells showed 
dominant expression of Endo H-resistant form of BCRP, which can be considered as complex-glycosylated (C-G) form that localizes in the golgi or PM (Fig. 1A, upper pannel). There were also a few expression of simply-glycosylated (S-G) form of BCRP in WTBCRP- transfected HEK293 cells as determined by Endo H and PNG-ase F digestions (Fig. 1A, upper pannel). Whereas N596Q BCRP, in which Asn596 is substituted by Gln596, preferentially undergoes ERAD because of the lack of N-linked glycosylation although its PM targeting and transporter activity are less affected if it is trafficked from the ER to PM [9]. We also checked the expression pattern of N596Q BCRP in HEK293 cells and confirmed the expression of non-glycosylated (Non-G) form of BCRP (Fig. 1A, lower pannel). Since ER is considered as an important organelle to determine the fate of these BCRP proteins, we first screened ER-localized factors that functions in the regulation of BCRP expression. Screening was performed by the change of BCRP expression level and pattern after the overexpression of ER-localized E3 ubiquitin ligases (WT gp78, WT Rma1, WT HRD1 and WT CHIP) and co-factor Derlin-1 that are previously shown to be involved in the degradation of several $\mathrm{ABC}$ transporters [17-19]. We also tried the overexpression of E3 activity-deficient dominant-negative mutant form of these E3 ligases (gp78 R2M, Rma1 C42S, HRD1 C329S and CHIP H260Q) in this study. Notably, none of WT and dominantnegative mutant form of E3 ligases did not robustly affect the expression level and pattern of both WT and N596Q BCRP (Fig. 1B-E), while overexpression of Derlin-1 strongly reduced $81 \mathrm{kDa}$ band (possibly C-G form) and increased $72 \mathrm{kDa}$ band (possibly S-G form) of WT BCRP (Fig. 2F). More interestingly, Derlin-1 overexpression strongly inhibited the expression level of N596Q BCRP (Fig. 2G). Thus, these data suggest that Derlin-1, but not other known E3 ligases tested, may be the critical molecule to control the expression of both WT and N596Q BCRP proteins.

\subsection{Exogenous Derlin-1 negatively regulates WT BCRP expression via inhibition of ER-to- Golgi transport of WT BCRP}

Having demonstrated that Derlin-1 overexpression dominantly increased the $72 \mathrm{kDa}$ band of WT BCRP protein, we next evaluated the origin of this dominant band. The $72 \mathrm{kDa}$ band was sensitive to Endo H digestion, confirming that Derlin-1 strongly increased S-G form of BCRP expression (Fig. 2A). Since decreased C-G form of BCRP was also accompanied with an increased expression of S-G form during Derlin-1 overexpression, we sought to determine whether ER-to-Golgi transport of WT BCRP is affected in Derlin-1-transfected HEK293 cells. As shown in Fig. 2B, treatment of brefeldin A (BFA), a ER-to-Golgi transport inhibitor [20], increased accumulation of ER form of WT BCRP (S-G) (pcDNA3.1, $0 \mathrm{~min}$ ) and this band was gradually converted to $\mathrm{C}-\mathrm{G}$ form of BCRP after the removal of BFA from culture media (BFA wash out). On the other hand, ER-to-Golgi transport of WT BCRP was significantly inhibited in the presence of exogenous Derlin-1 (Fig. 2B and C). Furthermore, WT BCRP-GFP expression was strongly limited in the ER in the presence but not the absence of exogenous Derlin-1 (Fig. 2D) and C-G form of WT BCRP-GFP was also converted to S-G form during Derlin-1 overexpression (Fig. 2E). Since interaction was observed between WT BCRP-GFP and Derlin-1 in the condition of Derlin-1 overexpression (Fig. 2F), these data suggest that Derlin-1 suppresses the maturation of WT $\mathrm{BCRP}$ protein possibly through their direct interaction. 
To exclude the possible role of Derlin-1 in regulating degradation of WT BCRP, protein stability in Derlin-1-transfected cells was evaluated by the cycloheximide (CHX) chase experiment. As shown in Fig. 2G and H, PNGase F-treated (+) WT BCRP expression, which represents total amount of WT BCRP protein, was minimally affected in Derlin-1transfected cells during the CHX chase period. Moreover, knockdown of Derlin-1 by siRNA did not have any impact on the expression of WT BCRP (Fig. 2I), suggesting that endogenous Derlin-1 may not function as a regulator of WT BCRP expression. Taken together, our data imply that exogenous but not endogenous level of Derlin-1 could negatively regulate the WT BCRP expression via the inhibition of ER-to Golgi transport of WT BCRP.

\subsection{Derlin-1 facilitates ERAD of N596Q BCRP protein via reduction of its protein stability}

Since Derlin-1 overexpression suppressed expression of N596Q BCRP protein (Fig. 1G), we hypothesized that Derlin-1 could facilitate ERAD of N596Q BCRP protein. Treatment with MG-132, a proteasome inhibitor [21], caused increased expression of N596Q BCRP protein, which is consistent with the previous study that showed that N596Q BCRP is relatively susceptible to ERAD (Fig. 3A) [9]. Interestingly, knockdown of Derlin-1 significantly increased the expression of N596Q BCRP protein to a similar extent as MG-132 treatment (Fig. 3B and C), implying the possible involvement of Derlin-1 in the regulation of N596Q BCRP degradation. Accordingly, significant reduction of N596Q BCRP expression was observed in the presence of Derlin-1 after $6 \mathrm{~h}$ CHX chase period (Fig. 3D and E), suggesting that both endogenous and exogenous Derlin-1 could reduce stability of N596Q protein and may contribute to ERAD of N596Q BCRP.

\subsection{Derlin-1 could also facilitate ERAD of non-glycosylated form of WT BCRP}

Recent report demonstrated that N-linked glycan is important for stabilizing WT BCRP and that disruption of this linkage by tunicamycin, a N-linked glycosylation inhibitor, results in protein destabilization and enhanced ERAD [9]. Based on our finding that Derlin-1 facilitates ERAD of non-glycosylated form of BCRP protein, N596Q BCRP, it is quite reasonable to think that Derlin-1 is also involved in tunicamycin-induced degradation of WT BCRP. Consistently, tunicamycin converted the expression pattern of WT BCRP from C-G form to Non-G form in WT BCRP-transiently transfected- HEK293 cells (Fig. 4A) and therefore significantly reduced the amount of WT BCRP expression (Fig. 4B). Further, pretreatment with MG132 inhibited the effect of tunicamycin-dependent change of band patten and expression of WT BCRP (Fig. 4C), confirming the existence of tunicamycininduced degradation of WT BCRP in our system. Interestingly, significant inhibition of tunicamycin-induced degradation of WT BCRP was also observed by knockdown of Derlin-1 (Fig. 4D and E), suggesting that Derlin-1 could also facilitate ERAD of nonglycosylated form of WT BCRP.

\section{Discussion}

ER is a crucial organelle that controls the proper balance between the posttranslational events that promote productive folding and ERAD of newly synthesized secretory and integral membrane proteins including disease-associated $\mathrm{ABC}$ transporters $[8,22]$. Among 
these transporters, cystic fibrosis transmembrane conductance regulator (CFTR/ABCC7) is one of the best characterized $\mathrm{ABC}$ transporters in terms of the posttranslational quality control in the ER [22-24]. Accumulating evidences have demonstrated that several E3 ubiquitin ligases, such as CHIP, gp78 and Rma1, and its co-factor Derlin1 are important factors that control cellular homeostasis by facilitating the degradation of WT or mutant CFTR $[17,18,25]$. These results lead us to speculate on possible involvement of these factors for the posttranslational regulation of other ABC transporters including BCRP/ABCG2. Here, we identified Derlin- 1 as a vital factor that affects both glycosylated and nonglycosylated BCRP expression by different mechanisms (Fig. 4F). In the regulation of glycosylated WT BCRP, exogenously expressed Derlin- 1 seems to induce ER retention of BCRP protein by inhibiting ER to Golgi transport. On the other hand, Derlin-1 is likely to be an ERAD mediator of N-linked glycosylation-deficient mutant N596Q BCRP and tunicamycin-induced non-glycosylated WT-BCRP proteins.

It is still unclear how Derlin-1 regulates the expression of these BCRP proteins. Since physical interaction was observed between exogenously expressed Derlin-1 and WT BCRP protein (Fig. 2F), Derlin-1 may regulate WT BCRP trafficking through their direct interaction. However, no interaction was observed between endogenously expressed Derlin-1 and WT BCRP protein (Fig. 2F) and siDerlin-1 did not affect the WT BCRP protein status (Fig. 2I), implying that Derlin-1 functions as a regulator of WT BCRP only if it is overexpressed. Since there are only limited number of studies on the regulation of Derlin-1 expression, a causal relationship between Derlin-1-BCRP interaction and WTBCRP expression levels needs to be further investigated in many types of cells to explore the impact of their interaction. Meanwhile, our study also did not elucidate the mechanism responsible for the Derlin-1-induced non-glycosylated BCRP degradation. Increasing evidences suggest that Derlin-1 has a chaperone-like activity that allows it to function as a substrate selector for the specific E3 ligase complex such as Rma1 [18,25,26]. Notably, overexpression of WT form of Rma1 and HRD1 but not dominant negative form of Rma1 (C42S) and HRD1 (C329S) seemed to slightly decrease N596Q BCRP expression (Fig. 1C and D), implicating that Derlin-1 may work with Rma1 and/or HRD1 during nonglycosylated BCRP degradation. Further studies are needed to identify the co-factors with Derlin-1.

One key issue that has yet to be discussed is how Derlin-1 recognizes non-glycosylated BCRP as its substrate for ERAD. Whether it is the absence of glycosylation or of protein instability in the ER requires further study. In order to explore these possibility, we need to assess the effect of Derlin-1 knockdown on the expression of BCRP variant proteins which are known to undergo ERAD in a glycosylation state-independent manner. These include naturally occurring SNPs variants of BCRP such as Q141K, F208S and S441N [10,11]. Further, we could also assess the impact of Derlin- 1 on the expression of other BCRP variants such as R383A, G553L and G553E variants, which are also shown to be impaired N-linked glycosylation like N596Q BCRP [27,28]. Whether siDerlin- 1 stabilizes the above $\mathrm{BCRP}$ variant proteins is currently under investigation.

Overall, our data demonstrate that Derlin-1 interacts physically with WT BCRP and reduces its maturation, and Derlin-1 initiates the efficient ERAD of non-glycosylated WT and 
N596Q BCRP, indicating that Derlin-1 is the negative regulator for the expression of both glycosylated and non-glycosylated WT BCRP proteins. Although current study does not rule out the involvement of Derlin- 1 in the regulation of BCRP function such as transport activity, our results provide a novel posttranslational regulatory mechanism of BCRP by Derlin-1.

\section{Supplementary Material}

Refer to Web version on PubMed Central for supplementary material.

\section{Acknowledgments}

This work was supported in part by grants from the Ministry of Education, Science, Sport, and Culture (MEXT) of Japan and the Global COE Program (Cell Fate Regulation Research and Education Unit), MEXT, Japan.

\section{References}

1. Oldham ML, Davidson AL, Chen J. Structural insights into ABC transporter mechanism. Curr Opin Struct Biol. 2008; 18:726-733. [PubMed: 18948194]

2. Ni Z, Bikadi Z, Rosenberg MF, et al. Structure and function of the human breast cancer resistance protein (BCRP/ABCG2). Curr Drug Metab. 2010; 11:603-617. [PubMed: 20812902]

3. Kusuhara H, Sugiyama Y. ATP-binding cassette, subfamily G (ABCG family). Pflugers Arch Eur J Physiol. 2007; 453:735-744. [PubMed: 16983557]

4. Vlaming ML, Lagas JS, Schinkel AH. Physiological and pharmacological roles of ABCG2 (BCRP): recent findings in Abcg2 knockout mice. Adv Drug Deliv Rev. 2009; 61:14-25. [PubMed: 19118589]

5. Matsuo H, Takada T, Ichida K, et al. Common defects of ABCG2, a high-capacity urate exporter, cause gout: a function-based genetic analysis in a Japanese population. Sci Transl Med. 2009; 1:5ra11.

6. Noguchi K, Katayama K, Mitsuhashi J, et al. Functions of the breast cancer resistance protein (BCRP/ABCG2) in chemotherapy. Adv Drug Deliv Rev. 2009; 61:26-33. [PubMed: 19111841]

7. Yoh K, Ishii G, Yokose T, et al. Breast cancer resistance protein impacts clinical outcome in platinum-based chemotherapy for advanced non-small cell lung cancer. Clin Cancer Res. 2004; 10:1691-1697. [PubMed: 15014021]

8. Wakabayashi-Nakao K, Tamura A, Furukawa T, et al. Quality control of human ABCG2 protein in the endoplasmic reticulum: ubiquitination and proteasomal degradation. Adv Drug Deliv Rev. 2009; 61:66-72. [PubMed: 19111842]

9. Nakagawa H, Wakabayashi-Nakao K, Tamura A, et al. Disruption of N-linked glycosylation enhances ubiquitin-mediated proteasomal degradation of the human ATP-binding cassette transporter ABCG2. FEBS J. 2009; 276:7237- 7252. [PubMed: 19909340]

10. Furukawa T, Wakabayashi K, Tamura A, et al. Major SNP (Q141K) variant of human ABC transporter ABCG2 undergoes lysosomal and proteasomal degradations. Pharm Res. 2009; 26:469-479. [PubMed: 18958403]

11. Nakagawa H, Tamura A, Wakabayashi K, et al. Ubiquitin-mediated proteasomal degradation of non-synonymous SNP variants of human ABC transporter ABCG2. Biochem J. 2008; 411:623631. [PubMed: 18237272]

12. Knop M, Finger A, Braun T, et al. Der1 A novel protein specifically required for endoplasmic reticulum degradation in yeast. EMBO J. 1996; 15:753-763. [PubMed: 8631297]

13. Shimasaki S, Koga T, Shuto T, et al. Endoplasmic reticulum stress increases the expression and function of toll-like receptor-2 in epithelial cells. Biochem Biophys Res Commun. 2010; 402:235240. [PubMed: 20933500]

14. Taura M, Eguma A, Suico MA, et al. P53 regulates Toll-like receptor 3 expression and function in human epithelial cell lines. Mol Cell Biol. 2008; 28:6557-6567. [PubMed: 18779317] 
15. Sugahara T, Koga T, Ueno-Shuto K, et al. Calreticulin positively regulates the expression and function of epithelial sodium channel. Exp Cell Res. 2009; 315:3294-3300. [PubMed: 19799896]

16. Harada K, Okiyoneda T, Hashimoto Y, et al. Calreticulin negatively regulates the cell surface expression of cystic fibrosis transmembrane conductance regulator. J Biol Chem. 2006; 281:12841-12848. [PubMed: 16527813]

17. Ballar P, Ors AU, Yang H, et al. Differential regulation of CFTRDeltaF508 degradation by ubiquitin ligases gp78 and Hrd1. Int J Biochem Cell Biol. 2009; 42:167-173. [PubMed: 19828134]

18. Younger JM, Chen L, Ren HY, et al. Sequential quality-control checkpoints triage misfolded cystic fibrosis transmembrane conductance regulator. Cell. 2006; 126:571-582. [PubMed: 16901789]

19. Wang L, Dong H, Soroka CJ, et al. Degradation of the bile salt export pump at endoplasmic reticulum in progressive familial intrahepatic cholestasis type II. Hepatology. 2008; 48:15581569. [PubMed: 18798335]

20. Klausner RD, Donaldson JG, Lippincott-Schwartz J. Brefeldin A: insights into the control of membrane traffic and organelle structure. J Cell Biol. 1992; 116:1071-1080. [PubMed: 1740466]

21. Lee DH, Goldberg AL. Selective inhibitors of the proteasome-dependent and vacuolar pathways of protein degradation in Saccharomyces cerevisiae. J Biol Chem. 1996; 271:27280-27284. [PubMed: 8910302]

22. Nikles D, Tampe R. Targeted degradation of ABC transporters in health and disease. J Bioenerg Biomembr. 2007; 39:489-497. [PubMed: 17972020]

23. Amaral MD. CFTR and chaperones: processing and degradation. J Mol Neurosci. 2004; 23:41-48. [PubMed: 15126691]

24. Turnbull EL, Rosser MF, Cyr DM. The role of the UPS in cystic fibrosis. BMC Biochem. 2007; 8(Suppl 1):S11. [PubMed: 18047735]

25. Sun F, Zhang R, Gong X, et al. Derlin-1 promotes the efficient degradation of the cystic fibrosis transmembrane conductance regulator (CFTR) and CFTR folding mutants. J Biol Chem. 2006; 281:36856-36863. [PubMed: 16954204]

26. Bernardi KM, Williams JM, Kikkert M, et al. The E3 ubiquitin ligases Hrd1 and gp78 bind to and promote cholera toxin retro-translocation. Mol Biol Cell. 2010; 21:140-151. [PubMed: 19864457]

27. Polgar O, Ediriwickrema LS, Robey RW, et al. Arginine 383 is a crucial residue in ABCG2 biogenesis. Biochim Biophys Acta Biomembr. 2009; 1788:1434-1443.

28. Polgar O, Ozvegy-Laczka C, Robey RW, et al. Mutational studies of G553 in TM5 of ABCG2: a residue potentially involved in dimerization. Biochemistry. 2006; 45:5251-5260. [PubMed: 16618113]

\section{Appendix A. Supplementary data}

Supplementary data associated with this article can be found, in the online version, at doi: 10.1016/j.bbrc.2010.12.074. 
A

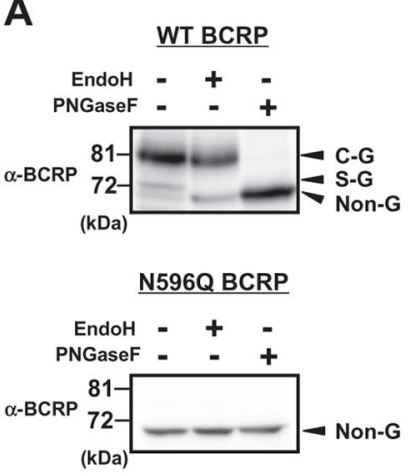

B

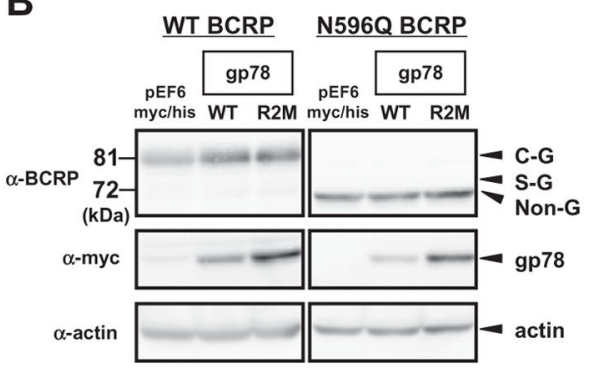

D

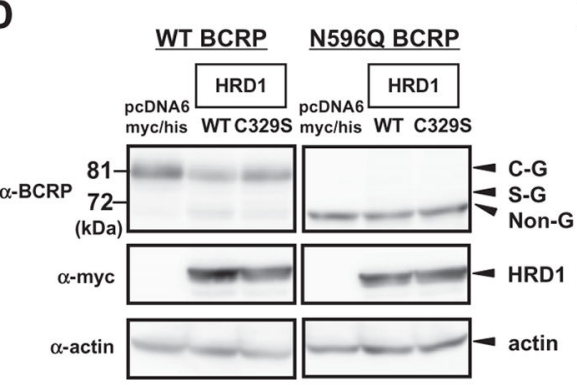

$\mathbf{E}$

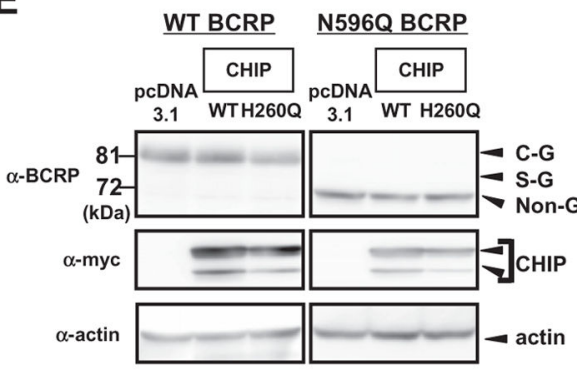

C

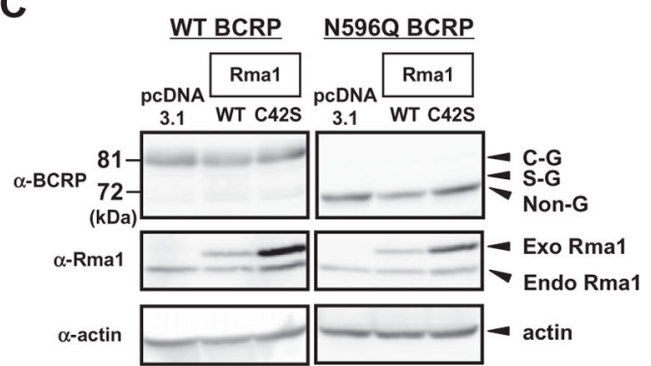

$\mathbf{F}$
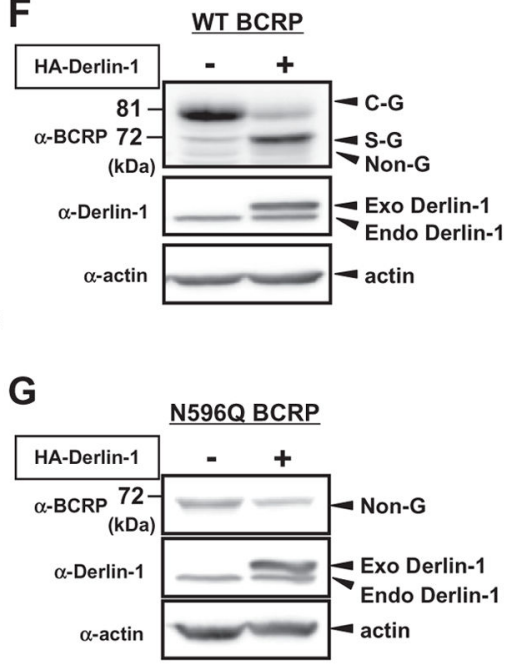

Fig. 1.

Derlin-1 is the critical molecule to control the expression of both WT and N596Q BCRP proteins. (A) HEK293 cells were transfected with WT or N596Q variant BCRP. Cell lysates were denatured and digested with Endo H or PNGase F. C-G, S-G and Non-G indicate complex-glycosylated, simply-glycosylated and non-glycosylated form of BCRP proteins, respectively. (B-E) WT or N596Q BCRP-transfected HEK293 cells were co-transfected with empty vectors or expression vectors that encode either WT or dominant-negative mutants of myc-tagged gp78 (B), Flag-tagged Rma1 (C), myc-tagged HRD1 (D) and myctagged CHIP (E). (F-G) Empty vector pcDNA3.1 or HA-tagged Derlin-1 was co-transfected with WT (F) or N596Q (G) BCRP in HEK293 cells. Exo-Derlin-1 and Endo Derlin-1 indicate exogenously and endogenously expressed Derlin-1, respectively. In all the experiments, whole cell lysates were subjected to Western blotting with the indicated antibodies. Actin was used as internal control. 
A

G
B

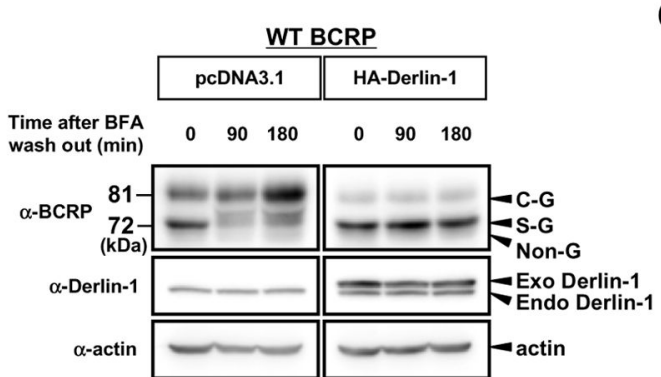

C

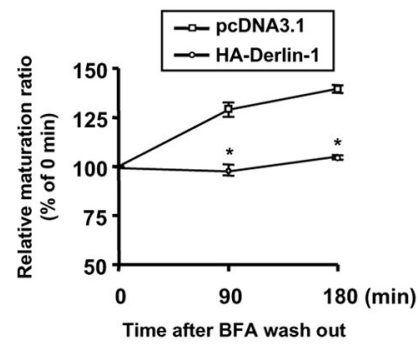

$\mathbf{E}$

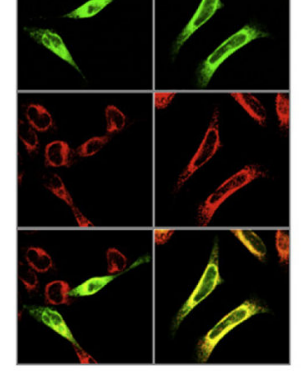

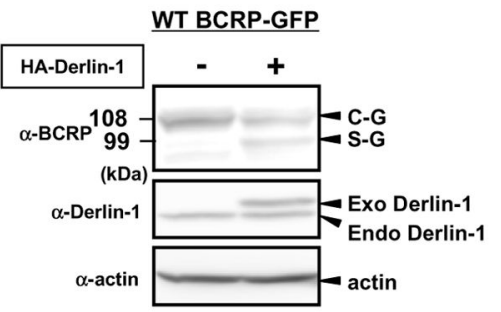

$\mathbf{F}$

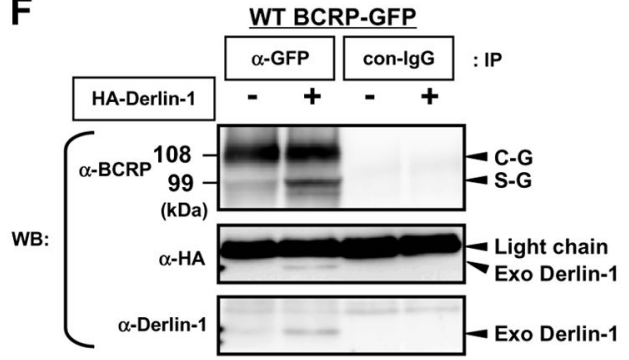

WT BCRP

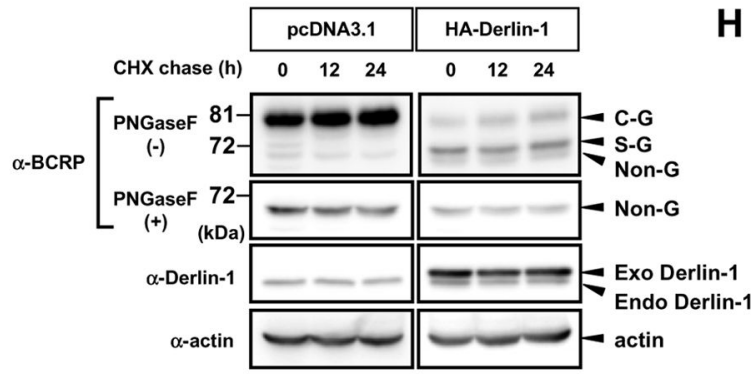

H

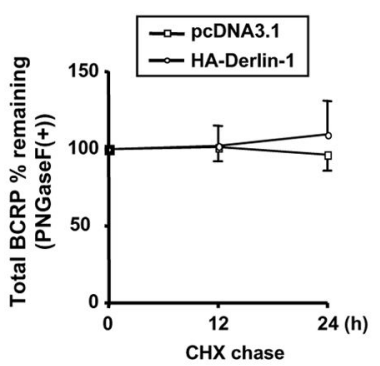

I

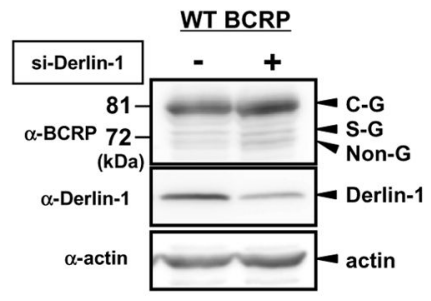

Fig. 2.

Derlin-1 negatively regulates WT BCRP expression via inhibition of ER-to Golgi transport of WT BCRP. (A) WT BCRP-transfected HEK293 cells were co-transfected with Derlin-1. Cell lysates were denatured and digested with Endo H or PNGase F, followed by Western blotting with anti-BCRP antibody. (B and C) WT BCRP-transfected HEK293 cells were cotransfected with empty vector pcDNA3.1 or HA-tagged Derlin-1. After transfection, cells were treated with $5 \mu \mathrm{g} / \mathrm{mL}$ of BFA for $6 \mathrm{~h}$ and then media was refreshed. Cells were harvested at 0,90 and 180 min after BFA wash out and subjected to Western blotting (B). Band intensities were measured by using Image Gauge software and relative maturation ratio was expressed as (intensity of C-G form)/(intensity of S-G form + intensity of C-G form) (C). ${ }^{*} P<0.05$ vs. pcDNA3.1-transfected cells. (D and E) Empty vector pcDNA3.1 or HA-tagged Derlin-1 was co-transfected into HeLa cells with GFP-tagged WT BCRP. Cells were fixed and localization of WT BCRP-GFP was determined by confocal laser scanning microscopy. To visualize ER, immunofluorescence analysis was performed with anti-KDEL antibody. (E and F) WT BCRP-GFP-transfected HEK293 cells were transfected with empty vector pcDNA3.1 or HA-tagged Derlin-1. Cells were lysed in digitonin buffer after transfection and lysates were subjected to Western blotting (E) and immunoprecipitation analysis (F). WT BCRP-GFP protein was immunoprecipitated by using anti-GFP antibody or non-immunized $\operatorname{IgG}$ (con-IgG) and immunoprecipitants were analyzed by Western 
blotting. (G and H) WT BCRP-transfected HEK293 cells were co-transfected with empty vector pcDNA3.1 or HA-tagged Derlin-1. Twenty-four hours after transfection, cells were treated with $50 \mu \mathrm{Mof} \mathrm{CHX}$ and harvested at 0,12 and $24 \mathrm{~h}$ after $\mathrm{CHX}$ treatment. Whole cell lysates were subjected to Western blotting (G). PNGase F-digested WT BCRP intensity was measured to express total BCRP amounts $(\mathrm{H})$. Result represents the mean + or -SEM performed. ${ }^{*} P<0.05$ vs. pcDNA3.1- transfected cells. (I) WT BCRP-transfected HEK293 cells were co-transfected with $50 \mathrm{nM}$ of control (-) or specific siRNA for Derlin-1 (+), followed by Western blotting. 
A

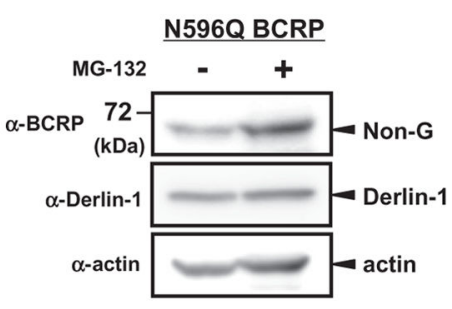

D

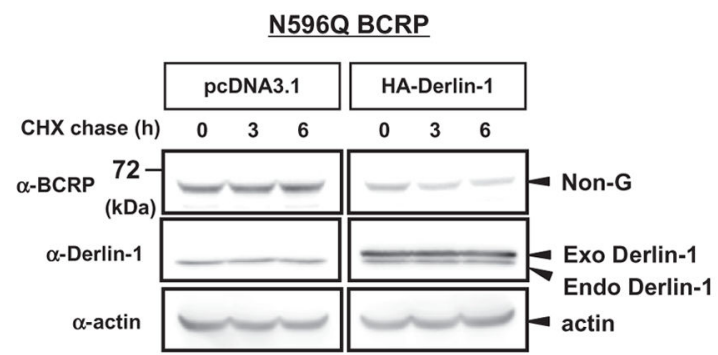

B

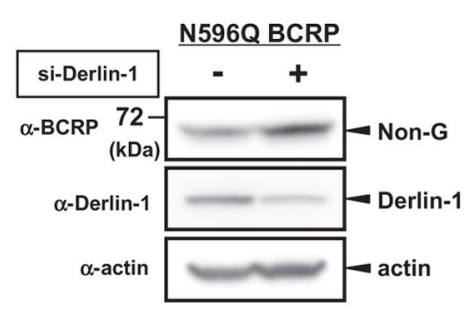

E

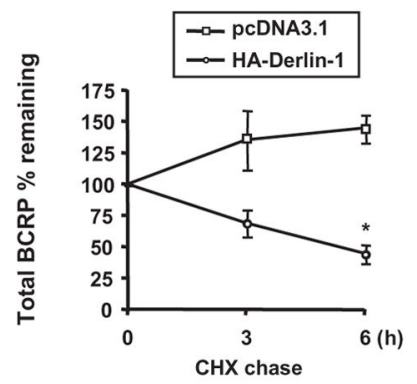

C

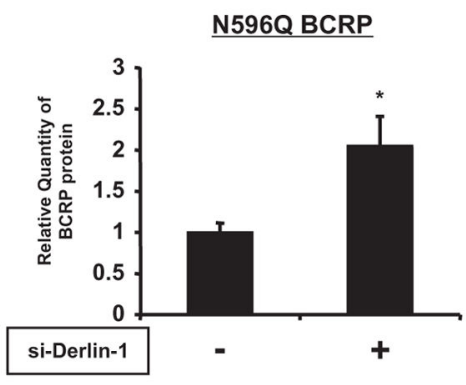

Fig. 3.

Derlin-1 facilitates ERAD of N596Q BCRP protein via reduction of its protein stability. (A) N596Q BCRP-transfected HEK293 cells were treated with $5 \mu$ M of MG132 for $24 \mathrm{~h}$ and lystaes were subjected to Westen blotting. (B and C) N596Q BCRP-transfected HEK293 cells were co-transfected with $50 \mathrm{nM}$ of control (-) or specific siRNA for Derlin-1 (+), followed by Western blotting (B). Band intensities were measured (C). ${ }^{*} P<0.05$ vs. control siRNA-transfected cells. (D, E) N596Q BCRP-transfected HEK293 cells were cotransfected with empty vector pcDNA3.1 or HA-tagged Derlin-1. After transfection, cells were treated with $200 \mu \mathrm{M}$ of $\mathrm{CHX}$ and harvested at 0,3 and $6 \mathrm{~h}$ after CHX treatment. Whole cell lysates were subjected to Western blotting (D). Non-G N596Q BCRP intensity was measured to express total BCRP amounts (E). ${ }^{*} P<0.05$ vs. pcDNA3.1- transfected cells. 
A

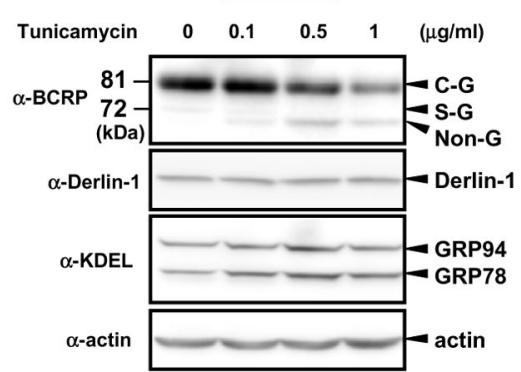

D

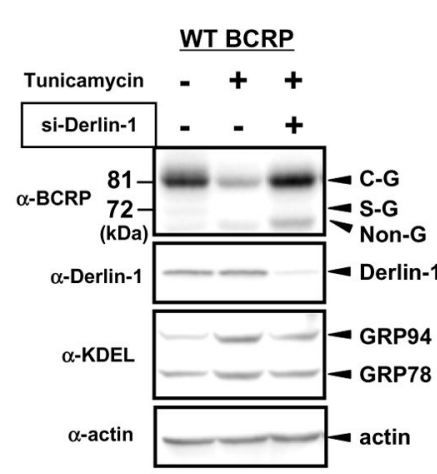

E
B

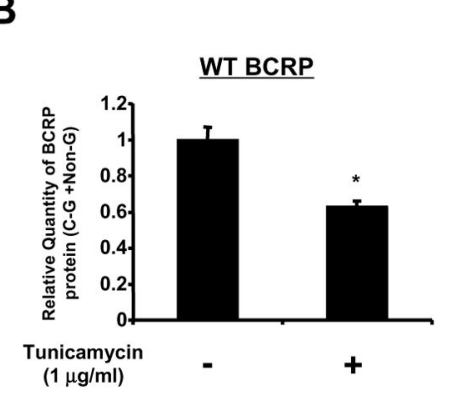

C

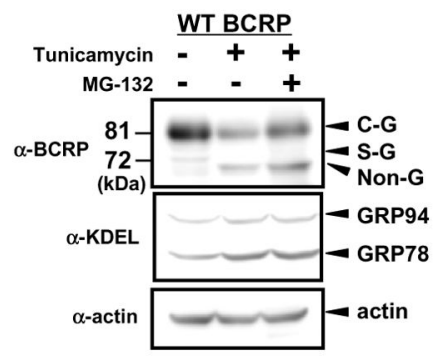

$\mathbf{F}$

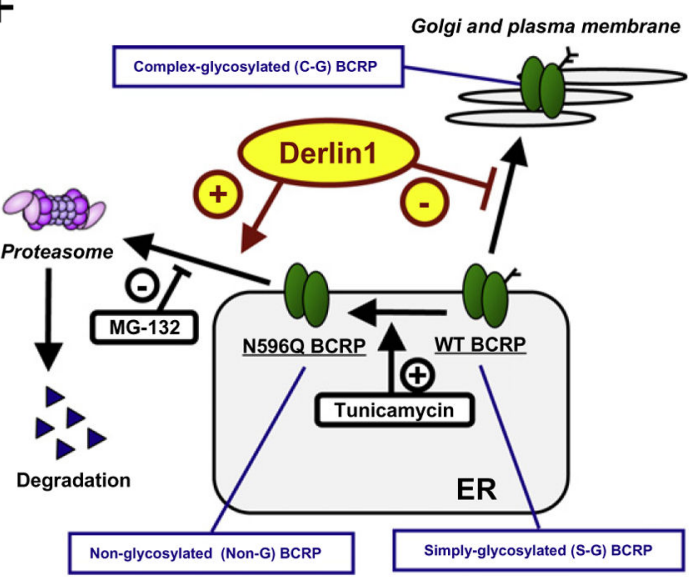

WT BCRP

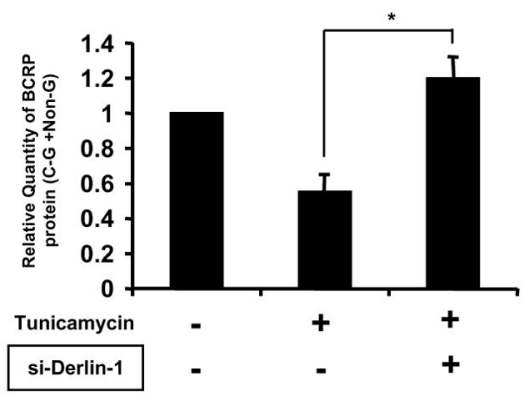

Golgi and plasma membrane

部

Fig. 4.

Derlin-1 also facilitates ERAD of non-glycosylated form of WT BCRP. (A) WT BCRPtransfected HEK293 cells were treated with various concentration of tunicamycin for $16 \mathrm{~h}$, followed by Western blotting. (B) Band intensities of tunicamaysin-treated samples (1 $\mathrm{g} / \mathrm{mL})$ were measured. Band intensities of (C-G WT BCRP)+(Non-G WT BCRP) were calculated to express total BCRP amounts. ${ }^{*} P<0.05$ vs. non-treated cells. (C) WT BCRPtransfected HEK293 cells were treated with $1 \mathrm{~g} / \mathrm{mL}$ of tunicamycin in the presence or absence of 5 M of MG-132 for $16 \mathrm{~h}$, followed by Western blotting. (D and E) WT BCRPtransfected HEK293 cells were co-transfected with $50 \mathrm{nM}$ of control (-) or specific siRNA for Derlin-1 (+). After transfection, cells were treated with $2 \mathrm{~g} / \mathrm{mL}$ of tunicamycin for $16 \mathrm{~h}$, followed by Western blotting. Band intensities of (C-G WT BCRP)+(Non-G WT BCRP) were calculated to express total BCRP amounts. ${ }^{*} P<0.05$ vs. tunicamycin-treated, control siRNA-transfected cells. (F) Schematic flow of Derlin1-dependent posttranslational regulation of glycosylated and non-glycosylated WT or N596Q BCRP. Derlin-1 physically interacts with WT BCRP and reduces its maturation, and Derlin-1 initiates the efficient ERAD of non-glycosylated WT or N596Q BCRP. Tunicamycin increases Non-G BCRP. MG-132 inhibits ERAD of Non-G WT-BCRP and N596Q BCRP. 